\title{
Evaluation of the mutual inductance of any conductor arrangements for determining the position
}

Wießner, F.

Rückerl, Ch.

Eichhorn, K. Fr.

FTZ Leipzig e.V.

Wächterstr. 13

04107 Leipzig
Plohmann, D.

Sensorik-Bayern $\mathrm{GmbH}$

Josef-Engert-Straße 9

93053 Regensburg

\section{Introduction}

The model of an inductive sensor for determining position is simulated in this paper. First the conductor element method [1] for any positioned finite conductor is expanded for these calculations. With this approach complex circuit structures union could be decomposed into polygons. A specific sensor model is considered, for simulations. In this model the mutual inductance is given as a function of the position of the transmitter coil. In a second step, the position of the transmitter coil is calculated by the mutual inductance. The position of the transmitter coil, is calculated from the mutual inductance outgoing from a firmly arranged system of receipt coils. Thus a reasonable inductive position sensor which can be simulated on the basis of this paper realised.

\section{Calculation of the mutual inductance}

The mutual inductance of two conductor loops $C_{1}$ and $C_{2}$ can be given in general with (1).

$\mathrm{M}_{12}=\frac{\mu}{4 \pi} \underset{\mathrm{C}_{1} \mathrm{C}_{2}}{\operatorname{fl}} \frac{\mathrm{d} \overrightarrow{\mathrm{s}}_{1} \mathrm{~d} \overrightarrow{\mathrm{s}}_{2}}{\left\|\overrightarrow{\mathrm{r}}_{1}-\overrightarrow{\mathrm{r}}_{2}\right\|}$

If the conductor loops are approximated by polygon trains, the circulation integral (1) is disassembled into a limited number of computable integrals. In this case the mutual inductance results in (2):

$$
\mathrm{M}_{12}=\sum_{\mathrm{n}} \sum_{\mathrm{m}} \mathrm{L}_{\mathrm{nm}}
$$

$\mathrm{m}$ : pieces of conductor loop $C_{1} / \mathrm{n}$ : pieces of conductor loop $C_{2}$

Equation (3) is valid for the mutual inductance of two limited conductor pieces as per figure 1.

$$
\mathrm{L}_{\mathrm{nm}}=\frac{\mu \cdot \cos \left(\delta_{\mathrm{nm}}\right)}{4 \pi} \int_{\mathrm{t}_{1}}^{\mathrm{t}_{2}} \int_{\mathrm{x}_{1}}^{\mathrm{x}_{2}} \frac{\mathrm{dxdt}}{\mathrm{R}_{\mathrm{nm}}(\mathrm{x}, \mathrm{t})}
$$

Function $R_{n m}(x, t)$ from (3) is the distance of the straight lines $x$ and $t$ on where both conductor pieces lie. In this case the integration borders $x_{1}, t_{1}, x_{2}$ and $t_{2}$ result from the beginning and the end of the conductor pieces (fig. 1). The mutual inductance between two parallel conductors is the easiest way for the solution of the equation (3).

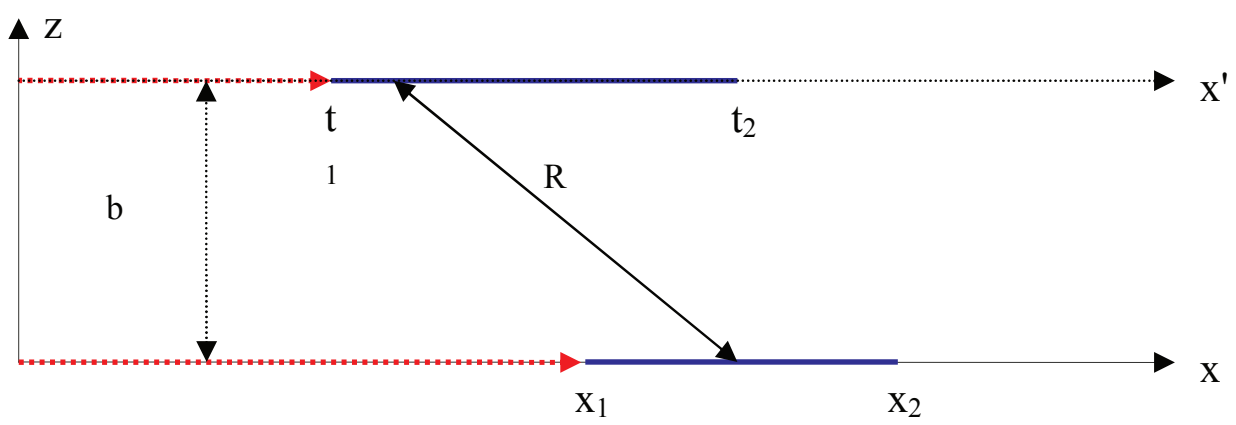

figure 1: Arrangement of parallel conductors 
In case of both conductor pieces running in parallel distance function is as follows(4):

$$
R=\sqrt{b^{2}+(t-x)^{2}}
$$

The analytic solution for the mutual inductance between two parallel conductors is given in [1] with (5).

$$
\mathrm{L}_{\mathrm{nm}}=10^{-7} \cdot \mu_{\mathrm{r}} \cdot \sum_{\mathrm{u}=1}^{2} \sum_{\mathrm{v}=1}^{2}(-1)^{\mathrm{u}+\mathrm{v}} \cdot\left\{-\left(\mathrm{x}_{\mathrm{v}}-\mathrm{t}_{\mathrm{u}}\right) \cdot \operatorname{arcsinh}\left(\frac{\mathrm{x}_{\mathrm{v}}-\mathrm{t}_{\mathrm{u}}}{\mathrm{b}}\right)+\sqrt{\mathrm{b}^{2}+\left(\mathrm{x}_{\mathrm{v}}-\mathrm{t}_{\mathrm{u}}\right)^{2}}\right\}
$$

For calculation of the mutual inductance of two, limited and twisted partial conductors it is supposed that both straight lines bearing the conductor pieces are in parallel levels to each other (fig. 2.). The connection of both straight lines who lie orthogonal to both levels is defined as a z-axis. Both direction vectors of the straight lines are the $x$ - and $y$-axis of the coordinate system. The dot product of both direction vectors is used for the determination of the angle $\delta$ between both straight lines.

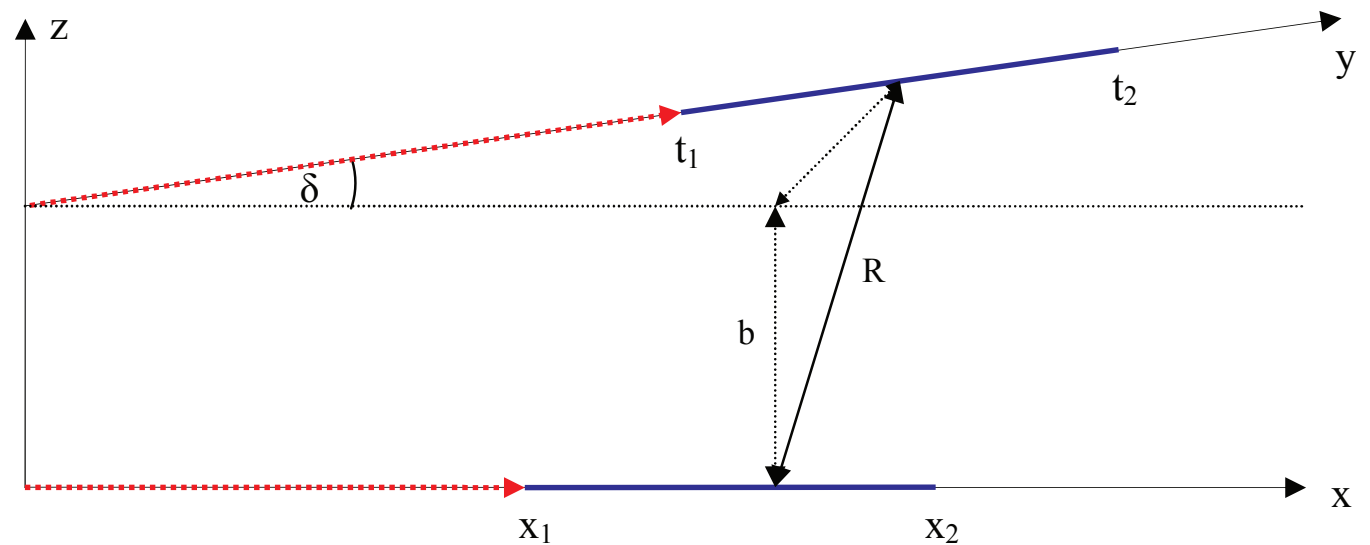

figure 2: Arrangement of twisted conductors

Value $b$ is the distance between both parallel levels in which the conductor pieces lie. This leads to distance function (6) of the twisted conductor pieces :

$$
\mathrm{R}=\sqrt{\mathrm{b}^{2}+\mathrm{x}^{2}+\mathrm{t}^{2}-2 \cdot \mathrm{t} \cdot \cos (\delta)}
$$

Distance function (6) for $\mathrm{R}_{\mathrm{nm}}$ delivers the mutual inductance delivers for twisted conductors (7).

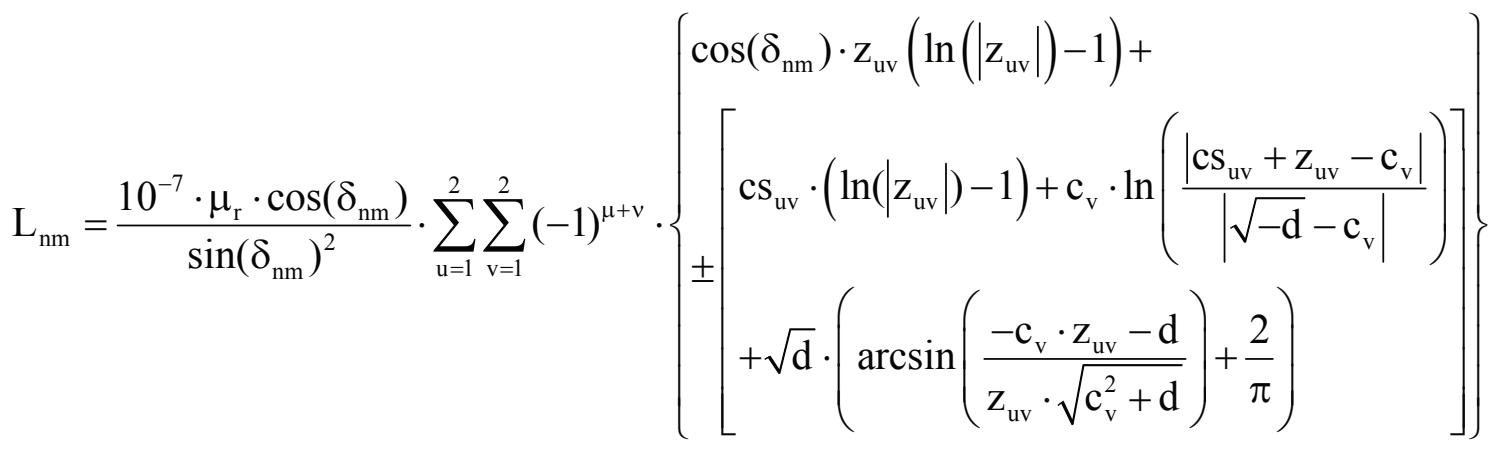

The following substitutions are used:

$$
\begin{aligned}
& \mathrm{d}=\mathrm{b}^{2} \cdot \sin ^{2}\left(\delta_{\mathrm{nm}}\right) \quad \mathrm{c}_{\mathrm{v}}=\mathrm{x}_{\mathrm{v}} \cdot \sin ^{2}\left(\delta_{\mathrm{nm}}\right) \quad \mathrm{cs}_{\mathrm{uv}}=\sqrt{\mathrm{z}_{\mathrm{uv}}{ }^{2}-2 \mathrm{c}_{\mathrm{v}} \mathrm{z}_{\mathrm{uv}}-\mathrm{d}} \\
& \mathrm{z}_{\mathrm{uv}}=\sqrt{\mathrm{t}_{\mathrm{u}}{ }^{2}+\mathrm{x}_{\mathrm{v}}{ }^{2}-2 \mathrm{x}_{\mathrm{v}} \mathrm{t}_{\mathrm{u}} \cos \left(\delta_{\mathrm{nm}}\right)+\mathrm{b}^{2}}-\mathrm{t}_{\mathrm{u}} \cdot \cos \left(\delta_{\mathrm{nm}}\right)+\mathrm{x}_{\mathrm{v}}
\end{aligned}
$$


The sign of the summation term of the equation of the mutual inductance (7) can be determined by solving the substitution variable $\mathrm{z}_{\mathrm{uv}}$ to the border of the double integral acts (6). This conversion of the root function delivers two solutions for border acts. The sign of the summation term in equation (7) is therefore, the sign of the root, corresponding with both borders.

Arbitrarily oriented conductors can be transferred by means of a coordinate transformation arranged similar to figure 2 , as the relative position of the conductor pieces does not change.

\section{Sensor model}

The model of the sensor arrangement is shown in figure 3. It includes a square transmitter coil(red), a square receiver coil(blue) and two equilateral, triangular receiver coil pairs placed opposite to each other. For this conductor loops with one turn are used. The transmitter coil is fed with a sine-ware alternating current which induces an alternating voltage in the receiver coil.
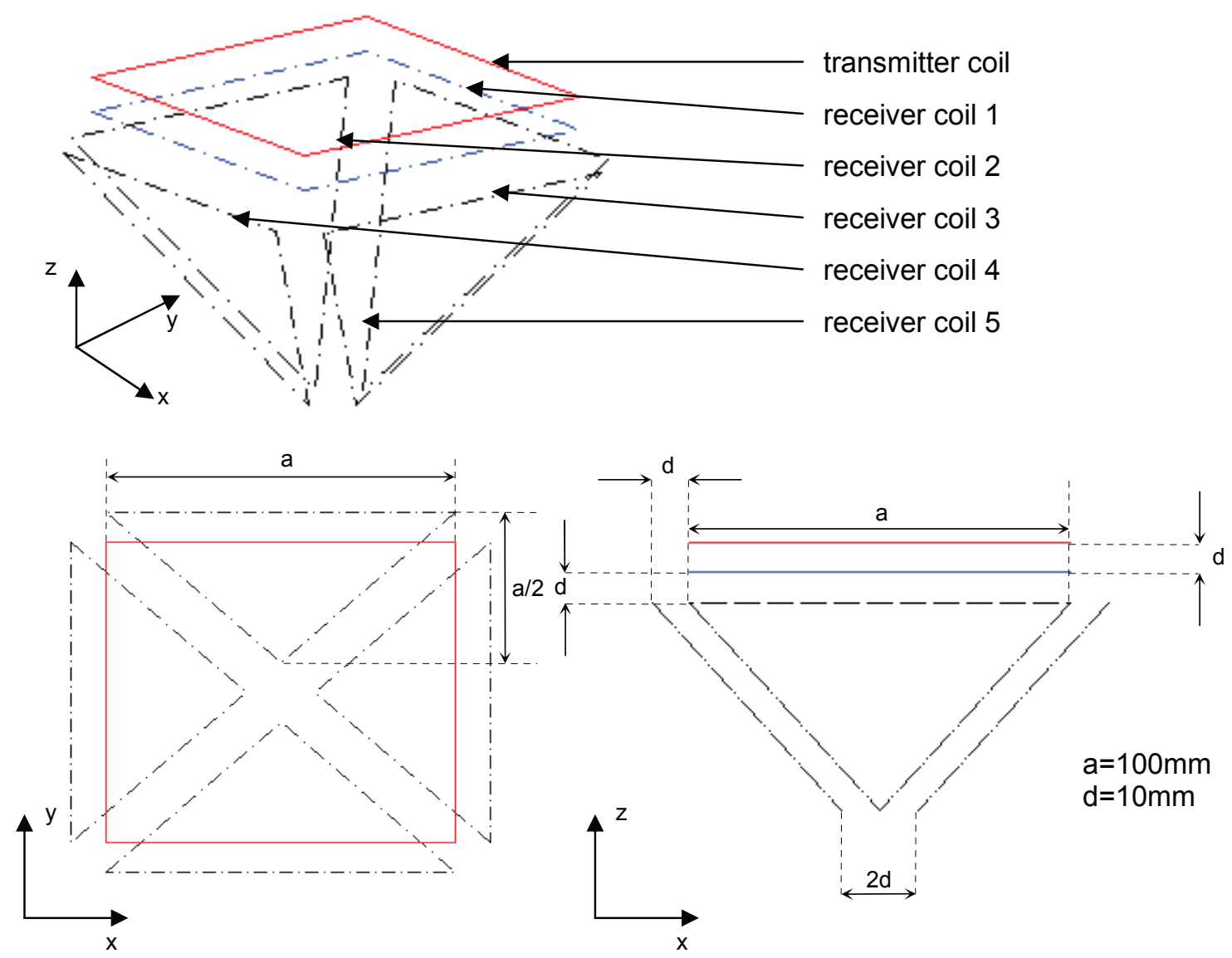

figure 3: Sensor model

The reactance forms the proportionality factor between the alternating current in the transmitter coil and the voltage induced in the receiver coils. The triangular receiver coil pairs are connected as difference coils. This leads on the one hand to a linearity of the measured voltage from the receiver coils and, on the other hand, to measuring values ranging around the zero point.

The receiver coil pair 2 and 3 are summarised in difference coil $M_{1}$. The receiver coils 4 and 5 are accordingly called difference coil $\mathrm{M}_{2}$.

\section{Solutions}

The simulation shows the values the mutual inductance (7) in dependence of the position of the transmitter. First the mutual inductance is shown in dependence of the movement in $x-y$-direction of the sensitive coil pairs. Conductor loops 2 and 3 are used as an $x$-sensitive sensor and conductor loop 4 and 5 as an y-sensitive sensor 

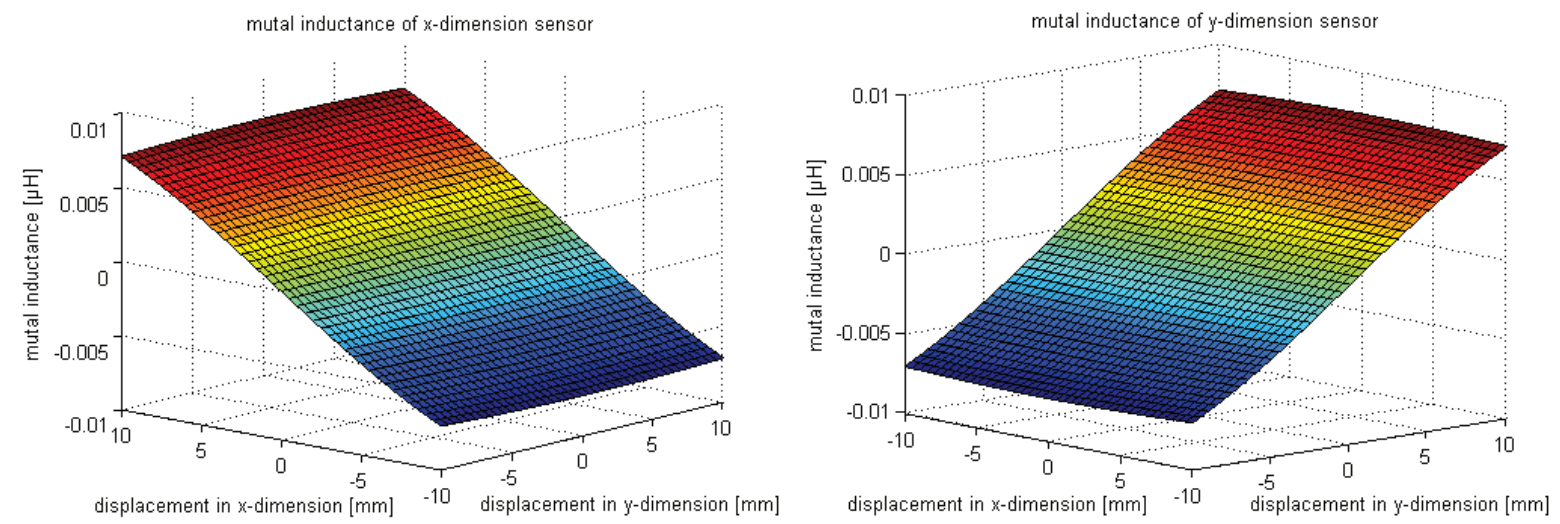

figure 4: Mutual inductance of the sensor arrangement for a movement in x-direction (left) and a movement in y-direction (right)

The mutual inductance of receiver coil 1 shows a strong dependence on the $x-y-$ movement of the transmitter coil (fig. 5).

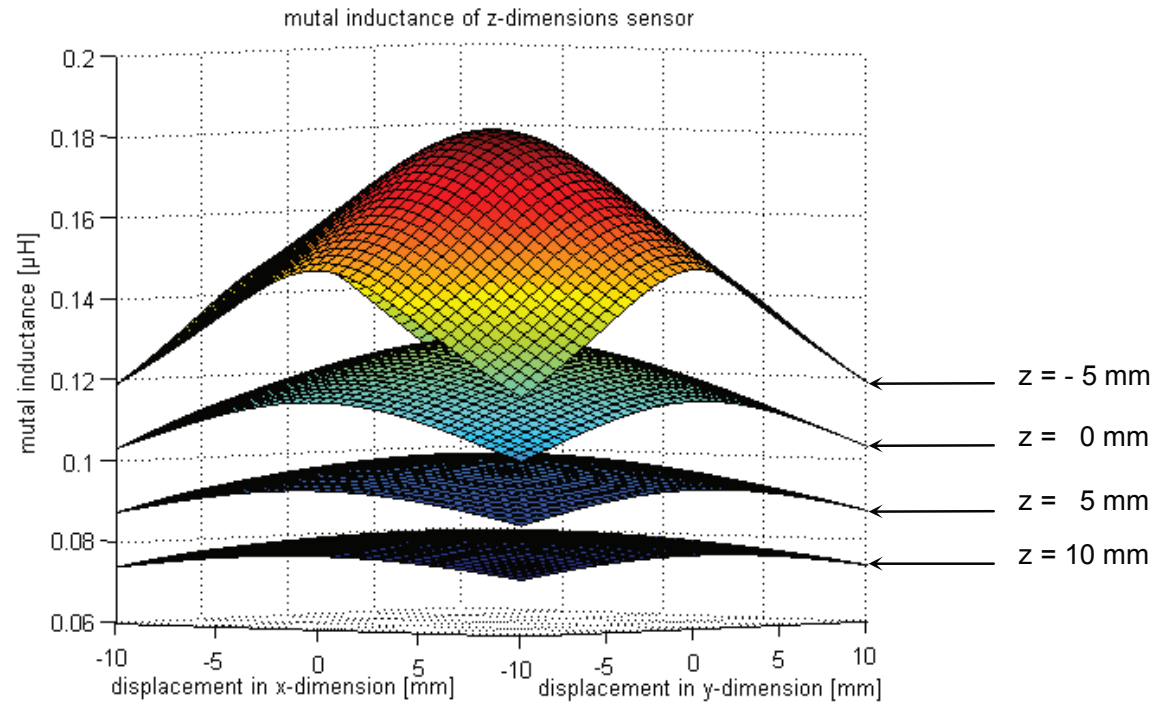

figure 5: Mutual inductance of the sensor arrangement for a displacement in z-direction with displacement in $\mathrm{x}$-y-direction
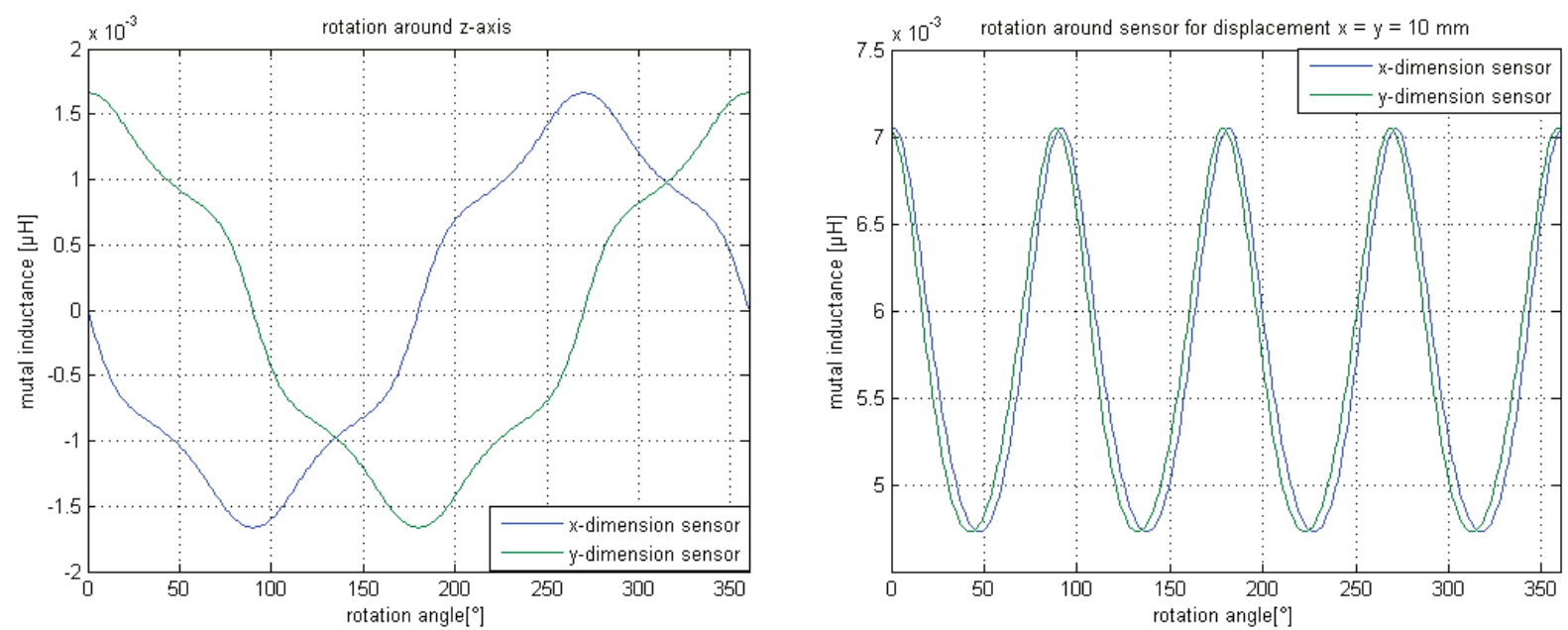

figure 6: mutual inductance of the sensor arrangement for a rotation around the $z$ axis (left) and one around itself (right)

Figure 6 (left) shows the mutual inductances $M_{1}$ and $M_{2}$ with the rotation of the transmitter around the zaxis if the transmitter coil has an inclination angle of two degrees compared with its normal position. Both curves are shifted by $90^{\circ}$ which can be explaned by the coil positions. 
Figure 6 (rigth) shows the mutual inductances $M_{1}$ and $M_{2}$ if the transmitter coil rotates around itself, after shifting $10 \mathrm{~mm}$ in both $\mathrm{x}$ - and $\mathrm{y}$-direction.

In order to calculate the $x-y$-movement from the calculated mutual inductances of two arranged as difference coils each sensors (receiver coil 2 and 3 and receiver coil 4 and 5 (fig.3)), a function of the mutual inductance is approximated in dependence of the $x-y$-movement for both sensors by polynominterpolation. As the sensors are nearly linear in their measuring direction, it is sufficient to use a polynom of first order without absolute term.

$$
\mathrm{M}_{1}(\mathrm{x}, \mathrm{y})=\mathrm{a}_{1}(\mathrm{y}) \cdot \mathrm{x} \quad \text { (8a) } \quad \mathrm{M}_{2}(\mathrm{x}, \mathrm{y})=\mathrm{a}_{2}(\mathrm{x}) \cdot \mathrm{y}
$$

It is possible to show these connections in a polynoun of higher order. This is very expensive and the inverse functions provide difficulties with case differentiations. The sensitivity is also depending on vertical movements. This influence on the sensitivity (for the $x$-movement $a_{1}(y)$ and for the $y$-movement $a_{2}(x)$ ) includes a square dependence and, hence, can be described with a polynoun of the second order.

$$
\mathrm{a}_{1}(\mathrm{y})=\left(\mathrm{b}_{1} \cdot \mathrm{y}^{2}+\mathrm{b}_{0}\right) \quad \mathrm{a}_{2}(\mathrm{x})=\left(\mathrm{c}_{1} \cdot \mathrm{x}^{2}+\mathrm{c}_{0}\right)
$$

The coefficients $b_{0}$ and $c_{0}$ show the sensitivity of the sensors without being influenced. The coefficients $b_{1}$ and $c_{1}$ consider changes of the measuring sizes. The influence of the measuring sizes is square and therefore equal for negative and positive directions. To determine the coefficients of the function of the mutual inductance $M_{1}$ the measuring vectors are formed. The measuring value vector $T_{1}$ (10a) consists of the $x$-movements, the measuring value vector $T_{2}$ consists of the square $y$-movements in the first column the second column consists of coefficients(1). The corresponding is valid for the mutual inductance $\mathrm{M}_{2}$ (10b).

$$
\mathrm{T}_{1}=\left(\begin{array}{c}
\mathrm{x}_{1} \\
\mathrm{x}_{2} \\
\vdots \\
\mathrm{x}_{\mathrm{n}}
\end{array}\right) \quad \mathrm{T}_{2}=\left(\begin{array}{cc}
\mathrm{y}_{1}{ }^{2} & 1 \\
\mathrm{y}_{2}^{2} & 1 \\
\vdots & \vdots \\
\mathrm{y}_{\mathrm{n}}{ }^{2} & 1
\end{array}\right)
$$

$$
\mathrm{T}_{4}=\left(\begin{array}{cc}
\mathrm{x}_{1}{ }^{2} & 1 \\
\mathrm{x}_{2}^{2} & 1 \\
\vdots & \vdots \\
\mathrm{x}_{\mathrm{n}}{ }^{2} & 1
\end{array}\right)
$$

The product of sensitivity $a_{1}(y)\left(a_{2}(x)\right)$ and the measuring value vector $T_{1}\left(T_{3}\right)$ provides a vector of the mutual inductance $M_{1}\left(M_{2}\right)$ in dependence of the $x-(y-)$ movement. These are overestimated equation systems which can be solved by means of pseudo-inverse $(+)[2](11)$.

$$
\mathrm{a}_{1}(\mathrm{y})=\mathrm{T}_{1}^{+} \cdot \mathrm{M}_{1}(\mathrm{x}, \mathrm{y}) \quad \mathrm{a}_{2}(\mathrm{x})=\mathrm{T}_{3}^{+} \cdot \mathrm{M}_{2}(\mathrm{x}, \mathrm{y})
$$

This procedure is also executed for all values of the influencing parameter. Resulting in the coefficient vectors $a_{1}(y)$ and $a_{2}(x)$. These form with the measuring vectors $T_{2}$ and $T_{4}$ also an overestimated equation system which is solved with the method of least squares. From it follow, the coefficients $b_{1}, b_{0}$, $\mathrm{c}_{1}$ and $\mathrm{c}_{0}(12)$.

$$
\left(\begin{array}{l}
\mathrm{b}_{1} \\
\mathrm{~b}_{0}
\end{array}\right)=\mathrm{T}_{2}^{+} \cdot \mathrm{a}_{1}(\mathrm{y}) \quad\left(\begin{array}{l}
\mathrm{c}_{1} \\
\mathrm{c}_{0}
\end{array}\right)=\mathrm{T}_{4}^{+} \cdot \mathrm{a}_{2}(\mathrm{x})
$$

The coefficients are applied in the equations of the mutual inductance (9), resulting in an equation system which can be solved for the movements in $\mathrm{x}$ - and $\mathrm{y}$-direction. In comparison to the measurable size the size of influence owns only one low influence on the mutual inductance. Hence, it is to be neglected possibly the influencing of the influencing. For this the average of the sensitivity (13) of the influencing measuring size is formed.

$$
\overline{a_{1}(y)}=\frac{a_{1}\left(y_{1}\right)+a_{1}\left(y_{2}\right)+\cdots+a_{1}\left(y_{n}\right)}{n} \quad \overline{a_{2}(x)}=\frac{a_{2}\left(x_{1}\right)+a_{2}\left(x_{2}\right)+\cdots+a_{2}\left(x_{n}\right)}{n}
$$


This prevents case differentiations while turning the calculation for the movement in $\mathrm{x}$ - and $\mathrm{y}$-direction in dependence of the mutual inductances $M_{1}$ and $M_{2}$. This allows to describe the $x$ - and $y$-movement in dependence of the mutual inductance (14).

$$
x\left(M_{1}, M_{2}\right)=\frac{{\overline{a_{2}(x)}}^{2} \cdot M_{1}}{{\overline{a_{2}(x)}}^{2} \cdot b_{0}+b_{1} \cdot M_{2}^{2}} \quad y\left(M_{1}, M_{2}\right)=\frac{{\overline{a_{1}(y)}}^{2} \cdot M_{2}}{{\overline{a_{1}(y)}}^{2} \cdot c_{0}+c_{1} \cdot M_{1}^{2}}
$$

Many approximations are used with setting up the equation of mutual inductance, depending on the movements in $x$ - and $y$-direction. It is therefore indispensable to check these approximations carefully. Figure 7 shows the calculated movement as function of the mutual inductance.
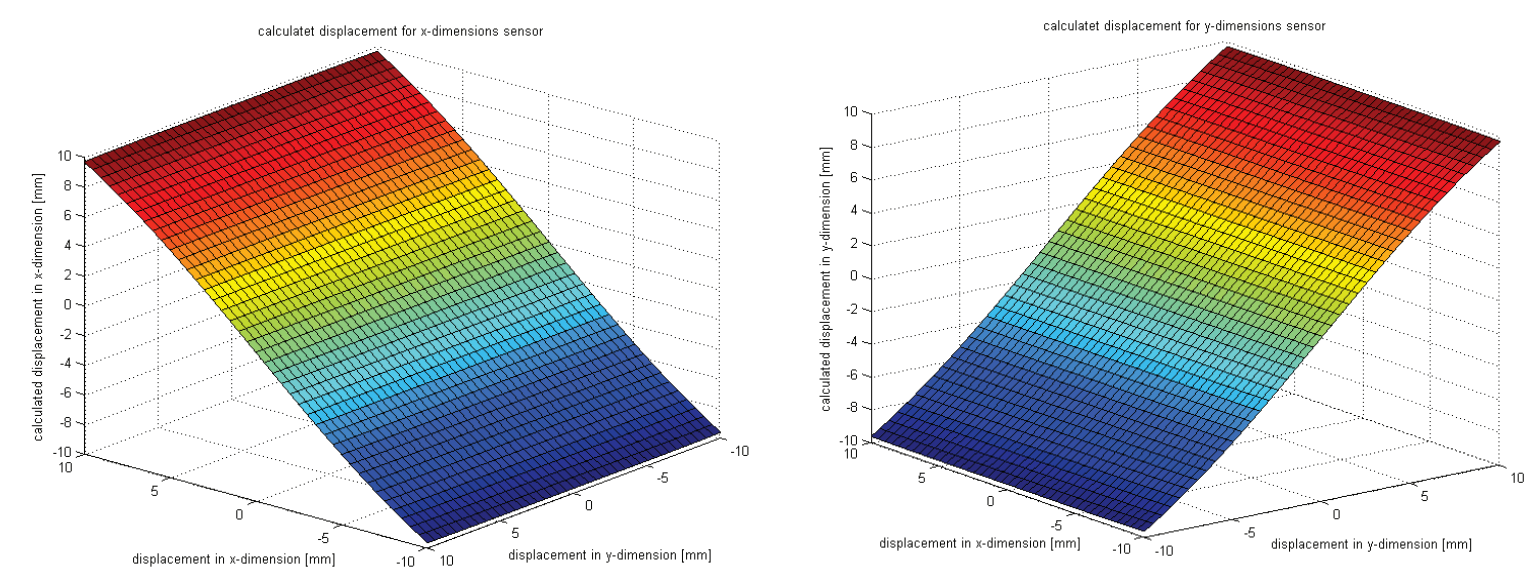

figure 7: calculated displacement(14) in dependence of the $x$-y-displacement for $x$ - direction (on the left) and y-direction (on the right)

It is obvious that the calculated values agree well with the initial values. The error caused by approximation is less than $3 \%$.

\section{Conclusion and outlook}

By extending the partial conductors method it is possible to calculate the mutual inductance of complicated conductor systems or coils. For this the conductors are fitted by the polygon trains. The mutual inductance is calculated between all partial conductors and all other conductors with the example of a position sensor the mutual inductances can be calculated as a function of the position of a transmitter coil and several receiver coils. This relation is reversed by means of approximation of the mutual inductance, thus to determine the position of the transmitter coil from the calculated mutual inductance. With this method divergences from linearity can be predicted as well methodical error e.g. twists of the sensor. Thus the introduced procedure is suitable for the optimization and interpretation of coil geometry.

\section{References}

[1] Eichhorn K.Fr.: Induktivitäten windschiefer Leitersysteme, Electrical Engineering, volume 61, number 6 , p. 353-362, Springer-Verlag, Erlangen, 1979

[2] Freund R.W.; Hoppe R.H.W.: Stoer/Bulirsch: Numerische Mathematik 1, 10. Edition, p. 318, Sringer -Verlag, Berlin, Heidelberg, New York, 2007 